\title{
Pediatric Ocular Trauma during Eid Festivities in Yemen
}

\author{
Tariek M Aldoais ${ }^{1,2}$, Mahfouth A Bamashmus ${ }^{3}$, Amal N Aldubhani ${ }^{2,3}$ \\ ${ }^{1}$ Ophthalmic Department, Al-Thawra Teaching Hospital, Sana'a, Yemen \\ ${ }^{2}$ Pediatric Unit, Magrabi Eye Hospital, Sana'a, Yemen \\ ${ }^{3}$ Eye Department, Faculty of Medicine and Health Sciences, Sana'a University, Sana'a, Yemen
}

Purpose: We sought to elucidate causes of ocular trauma in children younger than 17 years of age during the Eid festivities in Sana'a, Yemen.

Methods: A prospective observational case series study was conducted in two tertiary emergency hospitals (Al-Thawra and Magrabi Hospital). The Eid Al-Fitr festive holidays in 2016 lasted 10 days from July 3 to 12 . All children up to 17 years of age presenting with ocular trauma were included in the study. All patients underwent detailed history-taking and examination concerning ocular trauma.

Results: One hundred sixty children presented to the two hospitals with ocular injury. All were males (100\%). The mean \pm standard deviation age was $9.59 \pm 4.92$ years (range, $4-15$ years). The injury involved the right eye in 84 children (52.5\%) and the left eye in $76(47.5 \%)$ children. The majority of injuries $(n=152,95.0 \%)$ had occurred in the street, while eight (5.0\%) had happened at home. The most frequent cause of injury was toy guns and fireworks, while the most frequent findings were hyphema, corneal laceration, and corneal abrasion.

Conclusions: The causes of ocular injuries are diverse and tend to vary by geographical area. In particular, their incidence can increase during holidays, especially long ones. Usually, eye injuries with toy guns result in significant trauma that requires medical intervention and hospitalization with potential long-term eye complications. Public health workers need to conduct health education program to alert parents and health professionals to the danger of such toys.

Key Words: Festival holiday, Fireworks, Hyphema, Toy guns, Yemen

Ocular trauma is an important cause of acquired unilateral visual impairment in children in Yemen [1,2] and worldwide [3,4] and is the leading cause of non-congenital unilateral blindness [2]. Approximately $20 \%$ to $50 \%$ of eye injuries occur in the pediatric age group [5,6]. Eye trauma and visual impairment as a result of ocular trauma have a direct effect on society, leading to a variety of socioeco-

Received: October 22, 2019 Final revision: January 19, 2020

Accepted: February 14, 2020

Corresponding Author: Mahfouth A Bamashmus, FRCSEd, FRCOphth. Eye Department, Faculty of Medicine and Health Sciences, Sana'a University, PO Box 19576, Sana'a, Yemen. Tel: 967-733270277, Fax: 9671210021,E-mail: bamashmus@hotmail.com nomic problems, with a particularly devastating impact in children $[7,8]$. Ocular injuries can eventually lead to limitations in vision, unwanted cosmetic appearances, and psychosocial problems [9]. Ocular trauma often is usually accidental and affects mainly boys $[1,3]$.

Ocular injuries in children are usually monocular and often preventable [10]. To date, very few studies have been conducted on ocular trauma in Yemen [1]. Trauma associated with toy guns in the pediatric age group appears to increase in Yemen especially during holidays (Eid Alfitr and Eid Aladha) [11]. The most common site of injury among children is usually in the street or at home as a result of mechanical trauma [12]. The main causes are toy 
guns, fireworks, and accidental injury. Some of these injuries are due to innocuous objects, but other injuries are the result of exposure to dangerous objects.

In Yemen, injuries attributed to toy guns and fireworks are main reasons for emergency admission to the hospital, especially during the Eid holiday season. Toy guns or airsoft guns shoot small, hard plastic pellets using air pressure or a spring mechanism similar to that of real machine guns and are usually manufactured and imported from China [13]. The air pressure may be compressed by a powerful spring or by repetitive pumping of air into a gas chamber.

These guns are very popular among children in Yemen as they mimic the real machine guns that are widely available in the Yemeni community. Yemen has been an unstable country for a long time, with many civil wars occurring during the last few decades, and children tend to mimic the life they experience [14]. There are no age restrictions on buying these toy guns, and they can be purchased at a relatively low price. These toys are falsely perceived among children and families as harmless.

We report 160 cases of ocular injury secondary to toy gun pellets and fireworks during an Eid holiday period in the capital city, Sana'a, seeking to draw attention to the associated serious eye injuries among children.

\section{Materials and Methods}

We conducted a prospective observational case series study in two tertiary emergency eye hospitals in in Al-Thawra Teaching Hospital and Magrabi Eye Hospital in Sana'a, Yemen. The Eid Al-Fitr holiday season in 2016 included a 10-day holiday from July 3 to 12 . All children up to 17 years old who experienced ocular trauma during this period were included in the present study, while patients with a history of prior trauma were excluded.

All cases were evaluated at the ophthalmic emergency rooms of the two hospitals, and a decision was made to admit the child for operation or treatment, with follow-up occurring in the ophthalmic outpatient clinic. These two eye emergency departments are the main referral centers in Sana'a.

Each child's history was taken by the resident including age, sex, symptoms, and type and time of ocular injury. Eye examinations including visual acuity testing, slit-lamp examination, and direct and indirect ophthalmoscopy were performed. Intraocular pressure was measured when possible. If the retina was not visible by ophthalmoscopy, the child was referred for ultrasound B-scan or orbital computed tomography scan. Each child's treatment profile and follow-up were evaluated. Ocular injuries were classified according to the classification system of the International Society of Ocular Trauma [15].

This study was performed according to the guidelines of the Declaration of Helsinki, and ethical approval was obtained from the ethical committee of Yemen Magrabi Hospital (2016/03). The risks of surgical intervention were fully explained to the patients or their guardians in accordance with the Declaration of Helsinki, and verbal informed consent was obtained for inclusion in the study.

\section{Results}

A total number of 160 children presented to the two tertiary hospitals with ocular injury during the study period. Of these, $100 \%$ were male. The mean \pm standard deviation age of the study group was $9.59 \pm 4.92$ years (range, $4-15$ years); 12 children $(7.5 \%)$ were younger than 5 years (Table 1$)$.

Table 1. Characteristics on presentation to the emergency room

\begin{tabular}{lc}
\hline Characteristics on admission & Number $(\%)$ \\
\hline Sex & $160(100)$ \\
Male & $0(0)$ \\
Female & \\
Age (yr) & $12(7.5)$ \\
$4-5$ & $97(60.6)$ \\
$6-10$ & $51(31.9)$ \\
$11-15$ & \\
Eye & $84(52.5)$ \\
Right & $76(47.5)$ \\
Left & \\
Place of injury & $152(95.0)$ \\
Street & $8(5.0)$ \\
Home & \\
Cause of injury & $139(86.9)$ \\
Toy gun pellet (beads) & $18(11.2)$ \\
Fireworks & $2(1.3)$ \\
Stone & $1(0.6)$ \\
Janbia (knife) & $115(71.9)$ \\
Hospital & $45(28.1)$ \\
Al-Thawra Teaching Hospital & \\
Magrabi Eye Hospital & \\
\hline
\end{tabular}


Ocular injury was reported in the right eye in 84 children $(52.5 \%)$ and in the left eye in $76(47.5 \%)$ children (Table 1). All injuries affected one eye per child, with no cases of bilateral occurrence. The majority of injuries $(\mathrm{n}=152$, 95.0\%) had occurred in the street, while eight $(5.0 \%)$ were reported to have happened at home. All children were treated in the emergency department with the exception of those who needed surgical intervention for a ruptured globe $(16,10.0 \%)$; these patients were admitted for one day and followed up in the ophthalmic outpatient clinic. Table 2 shows the visual acuity at the time of presentation in the 160 cases. Among the total 160 cases of pediatric ocular injury, 139 (86.9\%) had trauma related with toy gun pellet (bead) exposure, while 18 cases (11.2\%) were attributed to fireworks. No eye protection was worn by any of the children injured during the study period.

The most frequent finding was hyphema ( $\mathrm{n}=126$, $78.75 \%$ ), followed by corneal laceration (i.e., ruptured globe) $(\mathrm{n}=16,10.0 \%)$, corneal abrasion $(\mathrm{n}=5,3.13 \%)$, and limbal ischemia $(n=3,1.88 \%)$. Table 3 describes the findings reported on presentation, which included traumatic cataract, vitreous hemorrhage, conjunctival injection, iridodialysis, subconjunctival hemorrhage, and lid swelling.

\section{Discussion}

In Yemen, during the last few years, children have come to prefer to play with toys that mimic their real life [14]; thus, given that machine guns (kalashnikov) are owned by every Yemeni adult, toy guns are similarly available in every city and village across Yemen. These toys are cheap and affordable to most children and fire 5- to 6-mm plastic pellets propelled either by a spring or compressed air. The material used to make these pellets is a very hard, non-compressible plastic.

These toy guns represent a major problem in Yemen, and authorities are doing their best to prevent their import; however, because of continued civil war in Yemen and the absence of real authorities in many places, smugglers are able to bring them into the country, ignoring the danger that they may cause. In Yemen, these toy guns have caused substantial morbidity over the years and at each holiday, especially those that are Islamic Eid holidays, which extend from five to seven days in length and sometimes longer. The particular danger of these toys is that they are
Table 2. Visual acuity at presentation

\begin{tabular}{lc}
\hline Visual acuity & No. of patients $(\%)$ \\
\hline $20 / 20$ & $2(1.25)$ \\
$20 / 25$ & $1(0.62)$ \\
$20 / 30$ & $3(1.88)$ \\
$20 / 40$ & $2(1.25)$ \\
$20 / 50$ & $4(2.50)$ \\
$20 / 70$ & $5(3.13)$ \\
$20 / 100$ & $6(3.75)$ \\
$20 / 200$ & $1(0.62)$ \\
$20 / 400$ & $9(5.62)$ \\
Counting fingers $2 \mathrm{~m}$ & $23(14.38)$ \\
Counting fingers $1 \mathrm{~m}$ & $30(18.75)$ \\
Counting fingers $0.5 \mathrm{~m}$ & $11(6.88)$ \\
Hand movement & $31(19.38)$ \\
Perception of light & $10(6.25)$ \\
No perception of light & $1(0.62)$ \\
Uncooperative & $21(13.12)$ \\
Total & $160(100)$ \\
\hline
\end{tabular}

Table 3. Type of injury at presentation to the emergency room

\begin{tabular}{lc}
\hline Type of eye injury & No. of patients $(\%)$ \\
\hline Hyphema & $126(78.75)$ \\
Ruptured globe & $16(10.00)$ \\
Corneal abrasion & $5(3.13)$ \\
Limbal ischemia & $3(1.88)$ \\
Traumatic cataract & $2(1.25)$ \\
Vitreous hemorrhage & $2(1.25)$ \\
Conjunctival injection & $2(1.25)$ \\
Irido dialysis & $2(1.25)$ \\
Subconjunctival hemorrhage & $1(0.62)$ \\
Lid swelling & $1(0.62)$ \\
Total & $160(100)$
\end{tabular}

available to very young children without age restriction [16].

Direct ocular impact by a projectile can produce major damage to the anterior and posterior segments, especially if fired from a close distance. These injuries if involving the posterior segment have a poorer prognosis compared to anterior segment trauma. There have been some case series of pediatric ocular injury from toy guns published 
in Denmark ( $\mathrm{n}=33$ cases) [17], Egypt ( $\mathrm{n}=32$ cases) [18], Kuwait ( $\mathrm{n}=19$ cases) [13], and Finland ( $\mathrm{n}=15$ cases) [11].

The mean \pm standard deviation age in our case series was $9.59 \pm 4.92$ years (range, $4-15$ years), while in previous case series from Kuwait [13] and Denmark [17], the mean ages were 7.8 years and 13 years, respectively. In our case series, males accounted for $100 \%$ of cases, which is similar to findings from both studies from Kuwait [13] and Denmark [17]. The reason behind the significant male dominance is believed to be due to their adventurous and aggressive nature.

Toy gun injuries mostly cause hyphema, but some cases can involve ruptured globe and need emergent surgical intervention. Hyphema was observed in 129 (78.5\%) cases in this study. Traumatic hyphema is caused by any projectile agent that strikes the eye such as projectile toys, fireworks, rocks, balls, and human fists [19]. Hyphema in the anterior chamber after toy gun pellet injury is thought to be related to a tear at the iris or ciliary body due to a sudden dynamic shift that displaces the iris and lens, leading to a resultant fluid wave in the aqueous humor and vitreous.

The most common site of bleeding is the anterior aspect of the ciliary body [19]. The blood exits from the anterior chamber via the trabecular meshwork and the Schlemm canal or the juxtacanalicular tissue. Hyphema in uncomplicated cases lasts for five to six days. Unfortunately, some cases can experience an increase in intraocular pressure or corneal staining if the hyphema is severe and there is damage to other parts of the eye such as the iris or lens. This increase in intraocular pressure can occur in the early presentation phase or can become a chronic long-term complication [20]. Intraocular pressure needs to be monitored during the follow-up period.

Ruptured globe was noted in 16 cases (10.0\%) with no case of retinal tear or retinal detachment at presentation and was most likely to occur if the gun was in close proximity to the eye. A few cases developed corneal abrasion ( $\mathrm{n}$ $=5,3.13 \%)$, traumatic cataract $(\mathrm{n}=2,1.25 \%)$, iridodialysis $(\mathrm{n}=2,1.25 \%)$, or vitreous hemorrhage $(\mathrm{n}=2,1.25 \%)$.

Fortunately, most pediatric trauma is preventable by adopting simple measures. Increasing both literacy and health awareness is vital; along these lines, the authorities in the capital city of Sana'a realized the importance of awareness and education of the community and took the initiative during the last few Eid holidays to produce educational banners for placement in the main streets (Fig. 1).

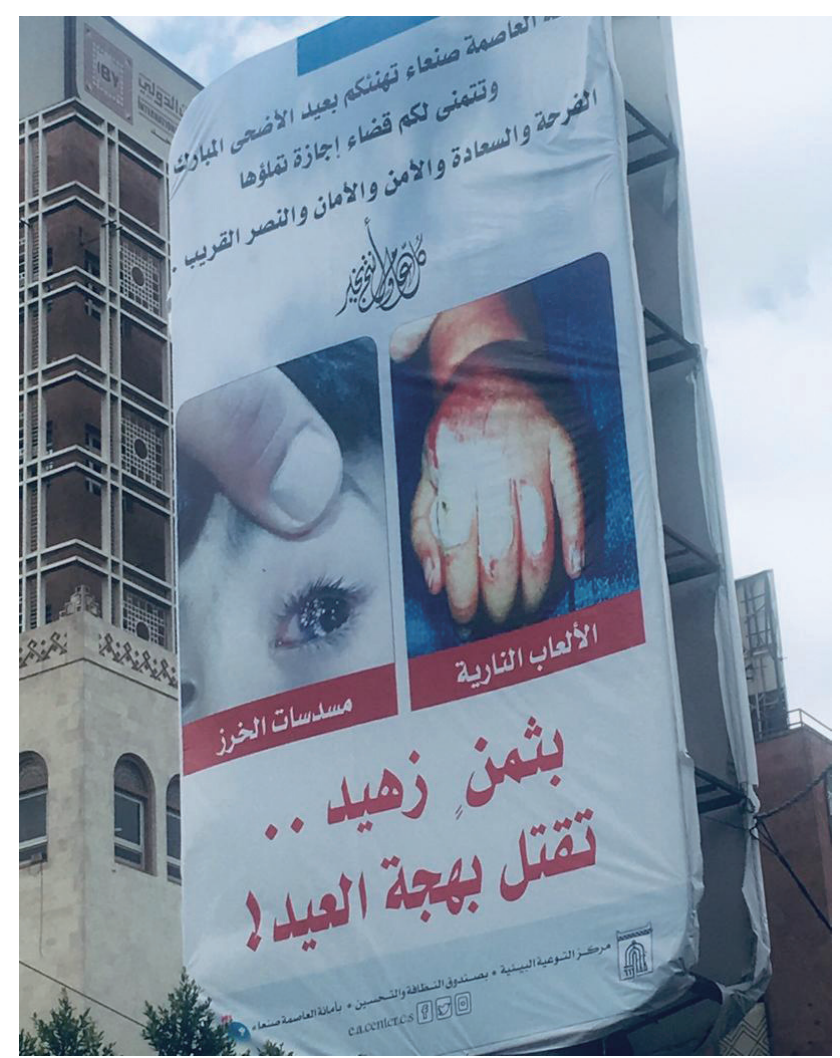

Fig. 1. Education banner regarding toy guns and fireworks. The banner says that Sana'a Capital Secretariat congratulate you for the Eid Al-Adha Festive season and wishes you to have a pleasant holiday and vacation full of joy, happiness, safety, security and near victory...for cheap money you buy fireworks and toy guns $\cdots$ you will kill the joy of the feast.

However, the irreversible nature of visual loss and the immense morbidity associated with these types of injuries need to be further emphasized and publicized.

In conclusion, toy guns and firework injuries are primary reasons for emergency admission to main Yemeni hospitals, especially during the Eid holiday season. There are no age restrictions to buy these toy guns in Yemen, helping make them very popular. Ocular trauma leads to visual impairment or even unilateral blindness, cosmetic blemishes, and resultant personality defects. The most important aspect of pediatric ocular trauma is prevention. Parents, teachers, and the community have an important role in prevention of such injuries. Playing with hazardous objects such as toy guns and fireworks should be discouraged. Appropriate management by a primary health care physician and general ophthalmologist, before ophthalmic consultation at a tertiary eye care center, is a key factor in preventing visual damage. 


\section{Conflict of Interest}

No potential conflict of interest relevant to this article was reported.

\section{Acknowledgements}

We thank administrators and staff of Althawra Teaching Hospital and Yemen Magrabi Eye Hospital for permitting us to conduct this study. They assisted and contributed in the patient's care in our study. Lastly, we appreciate the efforts and cooperation of all ophthalmic residents and patients they extended to us in this study. We specially thank Dr Hisham Al-Akhlee for his statistical help.

\section{References}

1. Bamashmus MA, Al-Shabooti A. Childhood eye injuries in Sana'a, Yemen. Middle East J Ophthalmol 2002;10:80-1.

2. Bamashmus MA, Al-Akily SA. Profile of childhood blindness and low vision in Yemen: a hospital-based study. East Mediterr Health J 2010;16:425-8.

3. Al Wadeai EA, Osman AA, Macky TA, Soliman MM. Epidemiological features of pediatric ocular trauma in Egypt. $J$ Ophthalmol 2016;2016:7874084.

4. Kuhn F, Morris R, Witherspoon CD, Mann L. Epidemiology of blinding trauma in the United States Eye Injury Registry. Ophthalmic Epidemiol 2006;13:209-16.

5. Soliman MM, Macky TA. Pattern of ocular trauma in Egypt. Graefes Arch Clin Exp Ophthalmol 2008;246:20512.

6. Podbielski DW, Surkont M, Tehrani NN, Ratnapalan S. Pediatric eye injuries in a Canadian emergency department. Can J Ophthalmol 2009;44:519-22.

7. Negrel AD, Thylefors B. The global impact of eye injuries. Ophthalmic Epidemiol 1998;5:143-69.

8. Thompson CG, Kumar N, Billson FA, Martin F. The aetiol- ogy of perforating ocular injuries in children. Br J Ophthalmol 2002;86:920-2.

9. Clarke A, Rumsey N, Collin JR, Wyn-Williams M. Psychosocial distress associated with disfiguring eye conditions. Eye (Lond) 2003;17:35-40.

10. Barry RJ, Sii F, Bruynseels A, et al. The UK Paediatric Ocular Trauma Study 3 (POTS3): clinical features and initial management of injuries. Clin Ophthalmol 2019;13:1165-72.

11. Haavisto AK, Sahraravand A, Puska P, Leivo T. Toy gun eye injuries: eye protection needed Helsinki ocular trauma study. Acta Ophthalmol 2019;97:430-4.

12. Brophy M, Sinclair SA, Hostetler SG, Xiang H. Pediatric eye injury-related hospitalizations in the United States. Pediatrics 2006;117:e1263-71.

13. Bouhaimed M, Alwohaib M, Alabdulrazzaq S, Jasem M. Toy gun ocular injuries associated with festive holidays in Kuwait. Graefes Arch Clin Exp Ophthalmol 2009;247:463-7.

14. Kalsvik S. Four years of brutal civil war in Yemen. Tidsskr Nor Laegeforen 2019;139.

15. Pieramici DJ, Au Eong KG, Sternberg P Jr, Marsh MJ. The prognostic significance of a system for classifying mechanical injuries of the eye (globe) in open-globe injuries. $J$ Trauma 2003;54:750-4.

16. Khalaily S, Tsumi E, Lifshitz T, et al. Airsoft gun-related ocular injuries: long-term follow-up. J AAPOS 2018;22:1079.

17. Saunte JP, Saunte ME. 33 Cases of airsoft gun pellet ocular injuries in Copenhagen, Denmark, 1998-2002. Acta Ophthalmol Scand 2006;84:755-8.

18. Shazly TA, Al-Hussaini AK. Pediatric ocular injuries from airsoft toy guns. J Pediatr Ophthalmol Strabismus 2012;49:54-7.

19. Rocha KM, Martins EN, Melo LA Jr, Moraes NS. Outpatient management of traumatic hyphema in children: prospective evaluation. J AAPOS 2004;8:357-61.

20. Ng DS, Ching RH, Chan CW. Angle-recession glaucoma: long-term clinical outcomes over a 10 -year period in traumatic microhyphema. Int Ophthalmol 2015;35:107-13. 